\title{
The Origin of the Universal Globular Cluster Mass Function
}

\author{
G. Parmentier ${ }^{1}$ and G. Gilmore ${ }^{2}$ \\ ${ }^{1}$ Argelander Institut fuer Astronomie, University of Bonn, Auf dem Huegel 71, D-53121 Bonn, \\ Germany \\ Scientific Research Worker of Fonds National de la Recherche Scientifique, Belgium \\ Humboldt Fellow \\ email: gparm@astro.uni-bonn.de \\ ${ }^{2}$ Institute of Astronomy, University of Cambridge, Madingley Road, Cambridge CB3 0HA, UK \\ email: gil@ast.cam.ac.uk
}

\begin{abstract}
Evidence favouring a Gaussian initial mass function for systems of old globular clusters has accumulated over recent years. We show that a bell-shaped mass function may be the imprint of expulsion from protoclusters of the leftover star forming gas due to supernova activity. Owing to the corresponding weakening of its gravitational potential, a protocluster retains a fraction only of its newly formed stars. The mass fraction of bound stars extends from zero to unity depending on the star formation efficiency achieved by the protoglobular cloud. We investigate how such wide variations affect the mapping of the protoglobular cloud mass function to the initial globular cluster mass function. We conclusively demonstrate that the universality of the globular cluster mass function originates from a common protoglobular cloud mass-scale of about $10^{6} \mathrm{M}_{\odot}$ among galaxies. Moreover, gas removal during star formation in massive gas clouds is highlighted as the likely prime cause of the predominance of field stars in the Galactic Halo.
\end{abstract}

Keywords. globular clusters: general, Galaxy: halo, Galaxy: formation, galaxies: star clusters

\section{Introduction}

The cluster mass function (i.e. the number of clusters per logarithmic mass interval $\mathrm{d} N / \operatorname{dlog} m$ ) is one of the primary characteristics of any globular cluster system hosted by a massive galaxy. Intriguingly, it proves to be almost independent of the size, the morphological type or the environment of the host galaxy. This universal globular cluster mass function is well fitted by a Gaussian with a mean of $\log \left(m / \mathrm{M}_{\odot}\right) \simeq 5.2$ and a standard deviation of $\sim 0.6$. Globular clusters having evolved over a Hubble-time in their galactic environment, their initial mass function has remained controversial, with two competing hypotheses. It may have been a featureless power-law with a slope of $\sim-1$, the Gaussian function characteristic of old globular cluster populations then resulting from a purely evolutionary effect, namely, the preferential removal of the more vulnerable low-mass clusters (Fall \& Zhang 2001). Yet, the present-day mass function represents an equilibrium state so that the initial one may also have been a Gaussian similar to that today (Vesperini 1998). If so, the Gaussian shape is the fossil imprint of the cluster formation process, this holding the clue to the universality of the observed globular cluster mass function. Parmentier \& Gilmore (2005) and Vesperini et al. (2003) provide evidence for a Gaussian initial globular cluster mass function in the Galactic halo and in the giant elliptical M87, respectively. Theoretical support for a bell-shaped initial cluster mass function has been missing so far however (although see Kroupa \& Boily 2002). 
As a result of the high star formation efficiency $(S F E)$ required to form a bound cluster, the initial cluster mass function has often been postulated to mirror that of their gaseous progenitors. Actually, numerous studies (e.g. Hills 1980; Lada; Margulis \& Dearborn 1984; Fellhauer \& Kroupa 2005) point that star forming clouds must be better than $30-50 \%$ efficient in converting gas into stars to produce bound stellar clusters. The limited variations in the SFE (i.e., less than a factor of 3) may then guarantee that the initial mass function of the clusters is that of their parent clouds. However, following the dispersal of the residual star forming gas by the combined actions of stellar winds and supernova explosions, the newly formed stars suddenly find themselves in a shallower gravitational potential, resulting into either the escape of some of them or even the complete disruption of the protocluster. Therefore, the initial mass of a stellar cluster is not determined by the $S F E \epsilon$ only. It depends on the mass fraction of the cluster parent cloud which is turned into stars remaining bound after the dispersal of the gaseous component. Specifically, the cluster initial mass $m_{\text {init }}$ obeys

$$
m_{\text {init }}=F_{\text {bound }} \times \epsilon \times m_{\text {cloud }},
$$

where $m_{\text {cloud }}$ is the mass of the gaseous progenitor and $F_{\text {bound }}$ is the mass fraction of stars remaining bound after gas removal. The bound fraction $F_{\text {bound }}$ ranges from 0 (when the $\epsilon$ is smaller than a threshold value $\epsilon_{t h} \simeq 0.35$ ) up to 1 (see the solid line in the left panel of Fig. 1). As a result, the assumed mirroring effect between the mass function of the cluster forming clouds on the one hand and the initial cluster mass function on the other hand can no longer be taken for granted, the latter depending on the former and on gas removal through the variations in the quantity $F_{\text {bound }} \times \epsilon$. We now investigate how the initial cluster mass function differs with respect to the protoglobular cloud mass function as a result of gas removal.

\section{From a universal protoglobular cloud mass-scale to the universal Gaussian globular cluster mass function}

As a first step, we assume a power-law protoglobular cloud mass spectrum

$$
\mathrm{d} N \propto m^{\alpha} \mathrm{d} m
$$

with $\alpha \simeq-1.7$, as is observed for giant molecular clouds and their star forming cores in the Local Group of galaxies (e.g. Rosolowski 2005). Star forming regions are characterized by a range in their respective star formation efficiency $\epsilon$, so that the protoglobular cloud mass spectrum is convolved with an $\epsilon$ probability distribution function, which we describe by a decreasing power-law of slope $\delta$ and core $r_{c}$, that is:

$$
\wp(\epsilon)=\frac{\mathrm{d} N}{\mathrm{~d} \epsilon}=c_{1}\left(1+\frac{\epsilon}{r_{c}}\right)^{\delta}+c_{4} .
$$

The two parameters $c_{1}$ and $c_{4}$ are determined so as to satisfy the two following constraints: (1) the integration of the probability distribution over the range $\epsilon=[0,1]$ is unity, and the probability $\wp(\epsilon)$ is zero when $\epsilon=1$. The formation of a bound star cluster requires its gaseous progenitor to achieve $\epsilon>\epsilon_{t h}$, i.e., the local star formation efficiency must be greater than $\simeq 0.3-0.4$. On the scale of a galaxy, star formation proceeds inefficiently, so the global star formation efficiency may be of order a few per cent only. The core $r_{c}$ and the slope $\delta$ of the efficiency distribution $\wp(\epsilon)$ are thus bounded so that the mean star formation efficiency, namely, the mass fraction of gas converted into stars for an entire system of protoglobular clouds, is one per cent. 
Following the onset of supernova activity, the gas-embedded cluster gets exposed as its residual gas is removed. Not only does the protocluster lose its gaseous component, it also loses a fraction of its initial stellar mass. We account for this phase by matching each efficiency value $\epsilon$ to the corresponding fraction $F_{\text {bound }}$ of bound stars. The $F_{\text {bound }}$ vs. $\epsilon$ relation we are using is shown as the solid line in the left panel of Fig. 1. The initial mass $m_{\text {init }}$ of globular clusters is then derived following equation 1.1.

Finally, the initial globular cluster mass function is evolved up to an age of 13 Gyr with Baumgardt \& Makino 's (2003) equation 12, which they derived by fitting the results of a large set of $N$-body simulations taking into account the effects of stellar evolution, of two-body relaxation and of cluster tidal truncation. At that stage, we obtain the model goodness of fit by comparing the evolved globular cluster mass function and the Old Halo globular cluster mass function.

If the protoglobular cloud mass function is a featureless power-law, then the initial cluster mass function is a power-law with the same slope, by virtue of the mass-independence of the star formation efficiency $\epsilon$ and of the bound mass fraction $F_{\text {bound }}$. If the protoglobular cloud mass function shows a lower mass-limit in the form of a truncation, however, it evolves into a bell-shaped initial cluster mass function. We have discussed how the cluster initial mass function responds to variations in the input parameters of our model (see Parmentier \& Gilmore 2007). We have successively varied the slope $\alpha$ of the cloud mass spectrum, its lower and upper limits, the slope $\delta$ and the scale-length $r_{c}$ of the star formation efficiency distribution $\wp(\epsilon)$, and the efficiency threshold $\epsilon_{t h}$ required to retain a bound core of stars (equivalently the gas removal time-scale $\tau_{g r}$ measured in units of the protocluster crossing-time $\tau_{\text {cross }}$; see the solid/dashed curves in the left panel of Fig. 1). The turnover location is mostly sensitive to the lower limit $m_{\text {low }}$ of the protoglobular cloud mass spectrum. The observed universality of the turnover of the globular cluster mass function would therefore originate from a common value among galaxies for the lower mass limit of protoglobular clouds, possibly with second-order variations driven by differences in the slopes of the cloud mass spectrum, that of the efficiency distribution $\wp(\epsilon)$, as well as by differences in the gas removal time-scale. The right panel of Fig. 1 shows the specific case of a protoglobular cloud mass function truncated at $m_{\text {low }} \simeq 6 \times 10^{5} \mathrm{M}_{\odot}$ (solid line with plus signs), along with the corresponding initial and 13-Gyr old cluster mass functions (solid line with plain/open symbols, respectively). The latter matches the observed Old Halo globular cluster mass function, with an incomplete gamma function of $Q \simeq 0.1$.

The bottom panel of Parmentier \& Gilmore 's (2007) figure 7 illustrates the goodness of fit of the evolved globular cluster mass function for various model parameters. The probability distribution function for the star formation efficiency $\wp(\epsilon)$ (i.e. $\delta$ and $r_{c}$ ) is adjusted so that the present globular cluster mass fraction in the Galactic halo is $2 \%$, as observed. That panel implies that a power-law protoglobular cloud mass spectrum with a narrow mass range, e.g. $10^{6} \mathrm{M}_{\odot} \lesssim m_{\text {cloud }} \lesssim 2 \times 10^{6} \mathrm{M}_{\odot}$, leads to a good fit $(Q \simeq 0.1)$ of the modelled cluster mass function onto the observed one. This suggests that the presentday halo cluster mass distribution, which covers two decades in mass, may equally-well arise from a characteristic mass for the protoglobular clouds. In order to investigate this point more closely, we now assume that the protoglobular cloud mass function obeys a Gaussian. The best solution under that assumption is shown in the right panel of Fig. 1, along with the power-law case which we have just discussed (dashed curves with plus signs, filled symbols and open symbols represent the protoglobular cloud mass function, the initial and evolved globular cluster mass functions). A Gaussian protoglobular cloud mass function with a mean of $2 \times 10^{6} \mathrm{M}_{\odot}$ and a logarithmic standard deviation smaller than 0.4 provides a good fit to the Old Halo cluster mass function. 

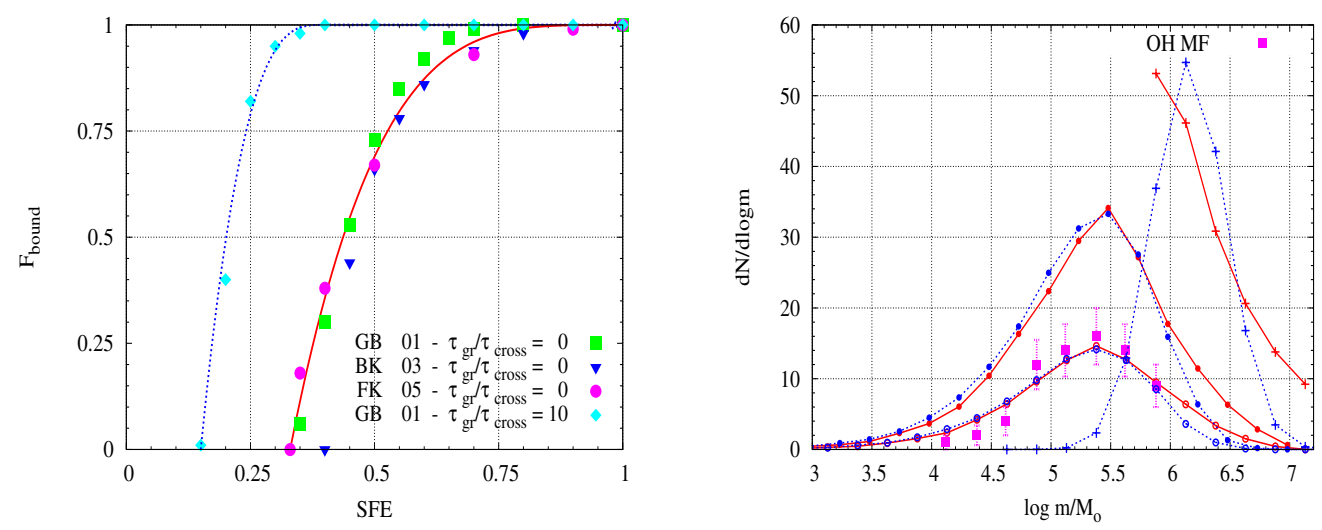

Figure 1. Left panel: Relations between the fraction $F_{\text {bound }}$ of stars remaining bound to the protocluster after gas removal and the star formation efficiency $\epsilon$ achieved by the gaseous progenitor. The solid/dashed lines correspond to the case of rapid/slow gas removal (i.e, $\tau_{g r}<<\tau_{\text {cross }}$ or $\left.\tau_{\text {gr }}>>\tau_{\text {cross }}\right)$. Data are from Geyer \& Burkert (2001), Boily \& Kroupa (2003) and Fellhauer \& Kroupa (2005) (respectively quoted as GB01, BK03 and FK05). Right panel: Initial/evolved (plain/open circles) cluster mass functions corresponding to two distinct mass functions for their gaseous progenitors: a power-law with a spectral index $\alpha=-1.7$ and truncated at a mass of $6 \times 10^{5} \mathrm{M}_{\odot}$ and a Gaussian mass function with a mean logarithmic mass of 6.15 and a standard deviation of 0.3 (solid/dashed curves with plus signs, respectively). The Old Halo globular cluster mass function is depicted by the full squares.

In order for our model to explain the universal Gaussian globular cluster mass function, the protoglobular clouds must thus be characterized by an almost invariant high-mass scale, either in the form of a lower truncation of the power-law cloud mass spectrum $\left(m_{\text {low }} \simeq 6 \times 10^{5} \mathrm{M}_{\odot}\right)$ or in the form of a high mean logarithmic mass of $\simeq 6.2$ if the clouds are distributed following a Gaussian in log-mass. Although that issue remains debated, various models for the formation of the first bound objects in the Universe actually suggest that globular cluster gaseous progenitors are characterized by that high a massscale (see Parmentier \& Gilmore 2007, their section 3.5, for an in-depth discussion). That narrow mass range is then broadened and turned into a bell-shaped cluster initial mass function by gas removal, while the $\simeq 10^{6} \mathrm{M}_{\odot}$ protoglobular cloud mass-scale guarantees that the turnover settles at the observed value.

\section{The origin of halo field stars}

In the present-day Galaxy, the formation of unbound stellar groups is the rule and not the exception. Most field stars in the Galactic disc likely originate from embedded clusters which either lost a fraction of their original members or were disrupted while emerging out of their natal clouds (Lada \& Lada 2003). It is likely that that paradigm also characterizes the formation of the stellar halo. Actually, $98 \%$ of its mass consists of field stars and so large a mass fraction cannot be accounted for by the secular evaporation and disruption of globular clusters over a Hubble-time, regardless of the shape of the cluster initial mass function (Parmentier \& Gilmore 2005).

The violent relaxation phase affecting protoclusters following the expulsion of their residual star forming gas constitutes a prime candidate to explain the origin of field stars in the Galactic halo, without conflicting with the well-accepted paradigm following which most stars form in clusters. In our fiducial cases of the right panel of Fig. 1, at an age of $13 \mathrm{Gyr}$, the total mass fraction of field stars $f_{F S}=98 \%$ in the halo arises mostly 
$\left(f_{F S}=91 \%\right)$ from cluster infant mortality, i.e. most halo field stars are given off by star forming regions whose efficiency $\epsilon$ is less than the star formation efficiency threshold $\epsilon_{t h}$.

It is worth noting that this may help understand why field stars and globular cluster stars sometimes show different patterns in their light element abundances. Chemical abundance anomalies observed in globular cluster stars are usually ascribed to the accretion onto their surface of stellar winds of intermediate mass stars ascending the asymptotic giant branch, a process made feasible by the dense stellar environment characteristic of globular clusters. In contrast, to the possible exception of binary stars, $a b$ initio field stars remain unaffected by this external pollution.

\section{Conclusions}

In this paper, we have presented detailed simulations highlighting how the mass function of protoglobular clouds evolves into that of gas-free bound star clusters as a result of the expulsion of the residual star forming gas due to supernova activity. Combining our model generating cluster initial mass functions with the cluster evolutionary model of Baumgardt \& Makino (2003), we have subsequently investigated which input parameters reproduce both the present-day mass function of the Old Halo clusters and their present-day mass fraction in the stellar halo. Our model naturally explains the universality of the globular cluster mass function among galaxies if the protogalactic era sets a characteristic mass of about $10^{6} \mathrm{M}_{\odot}$ for the protoglobular clouds independent of the host galaxy. We point however that our model still lacks a crucial ingredient, namely, the tidal field of the host galaxy since the $F_{\text {bound }}$ vs. $S F E$ relation we use has been derived in the case of isolated globular clusters. The tidal radius of a cluster depending on its mass, the tidal field may also contribute to the shape of the initial globular cluster mass function. Whether the turnover location is affected as well remains to be investigated.

\section{Acknowledgements}

This research was supported by a Marie Curie Intra-European Fellowship within the $6^{\text {th }}$ European Community Framework Programme. GP also acknowledges support from the Belgian Science Policy Office in the form of a Return Grant and the Alexander von Humboldt Foundation in the form of a Research Fellowship.

\section{References}

Baumgardt, H. \& Makino, J. 2003, MNRAS 340, 227

Boily, C. M. \& Kroupa, P. 2003, MNRAS 338, 665

Fall, S. M. \& Zhang, Q. 2001, ApJ 561, 751

Fellhauer, M. \& Kroupa, P. 2005, MNRAS 630, 879

Geyer, M. P. \& Burkert, A. 2001, MNRAS 323, 988

Hills, J. G. 1980, ApJ 225, 986

Kroupa, P. \& Boily, C. M. 2002, MNRAS 336, 1188

Lada, C. J., Margulis, M., \& Dearborn, D. 1984, ApJ 285, 141

Lada, C. J. \& Lada, E. A. 2003, ARA\& $\&$ 41, 57

Parmentier, G. \& Gilmore, G. 2005, MNRAS 363, 326

Parmentier, G. \& Gilmore, G. 2007, MNRAS 377, 352

Rosolowski, E. 2005, PASP 117, 1403

Vesperini, E. 1998, MNRAS, 299, 1019

Vesperini, E., Zepf, S. E., Kundu, A., \& Ashman, K.M. 2003, ApJ 593, 760 